EDITORIAL

\section{Moving Forward with Public Health in Canada}

\author{
Michael M. Rachlis, MD, MSc, FRCPC
}

$\mathrm{I}$ $\mathrm{n}$ recent years, SARS, BSE, and other communicable disease outbreaks have highlighted Canada's neglect of public health. The 2003 federal SARS report chaired by Dr. David Naylor noted that experts have advised governments for years of public health's problems but their warnings were disregarded. As a result, Dr. Naylor wrote, "there is much to learn from the outbreak of SARS in Canada - in large part because too many earlier lessons were ignored."

In this issue of the CJPH, Dr. Kumanan Wilson of the University of Toronto raises important questions about the governance of public health in Canada. Since Dr. Wilson submitted his article, the government of Canada has established the Public Health Agency of Canada and appointed its first chief public health officer. This commentary reviews the events of the past six months and discusses what we need to move forward.

\section{A new public health agency for Canada}

Ottawa's March 2004 budget allocated significant new funding for a Canadian Public Health Agency. The finance minister promised more funding in the future. In September 2004, the agency was officially launched. It is based in Winnipeg but will also have offices in Ottawa. The new agency will be connected to the rest of the country's public health services through six national collaborating centres and a to-be-established Pan-Canadian Public Health Network. At present, the public health agency's website advises that the Network could "complement and - if it proves effective - eventually subsume, certain of the existing mechanisms and arrangements for intergovernmental collaboration on public health matters." It is further suggested that the Network could serve as a forum for:

- Promoting dialogue on public health issues;

- Coordinating responses to public health emergencies;

- Developing national public health strategies;

- Facilitating the development of national standards and agreements on issues such as resource and data sharing, accreditation of health professionals; and

- Encouraging the development of centres of public health expertise across the country.

The Prime Minister announced the appointment of Dr. David Butler-Jones of Saskatchewan as the country's first chief public health officer on September 24, 2004. As recommended by Naylor's report, the new agency is at arm's length from Health Canada. Dr. Butler-Jones will report directly to the federal Minister of Health, the honourable Ujjal Dosanjh. This model allows the agency to contract directly with provincial and local public health agencies. It also permits the agency to have a flexible, private sector-like human resource policy which is needed to recruit and maintain staff.

The Liberals now have a minority government and will have to follow through on their promises. The economy has grown beyond the budget's projections, so money shouldn't be a problem. ${ }^{3}$ When combined with public health reform in Ontario and some other provinces, these circumstances open a large policy window for public health in Canada. However, as the ink dries on the federal legislation and regulations, politics will be as important as governance for the success of the new agency.

Associate Professor, Department of Health Policy Management and Evaluation, University of Toronto 13 Langley Avenue, Toronto, ON M4K 1B4, Tel: 416-466-0093, E-mail: michaelrachlis@rogers.com
Who has jurisdiction for public health in Canada?

As Dr. Wilson comments, public health is a matter of joint federal and provincial jurisdiction. Unfortunately, up until recently, the federal government has been remarkably silent in asserting its claims to the area.

The constitution gives the federal government authority over quarantine, criminal law, and interprovincial and international commerce. The constitution also gives the federal government authority to pass laws to ensure "peace, order, and good government". It is true, as Dr. Wilson notes, that the actual extent of these powers is ambiguous. But, like any powers, they atrophy with disuse.

The federal government also has its spending powers. The courts have reasserted the federal authority to spend its money as it wishes, even in areas of provincial jurisdiction like health care or social services. Without the spending power, there would be no medicare.

Finally, the federal government derives considerable authority from its international treaties and agreements. During the SARS outbreak, the World Health Organization (WHO) dealt with the federal government, not directly with the provinces or local public health agencies. The WHO travel advisory posted for Toronto had major economic implications for the whole country. It appears that even if our country somehow tolerates a confusion of mandates, international agencies will not. These events finally forced Canada's policy-makers to deal with the country's dysfunctional public health system.

\section{What do we need to move forward?}

The first steps towards reform are encouraging, but public health advocates will have to keep up the pressure to ensure effective implementation. The first priority must be more federal resources. While, in theory, the federal government has ample constitutional justification for a muscular public health role, in practice it must work with and through the provinces.

The federal government solved its deficit problems partly through cutbacks to provincial transfer payments. Twenty years of federal government cutbacks, through three administrations, has cultivated an environment of distrust between Ottawa 
and the provinces. One current reality of Canadian political life is that the provinces require the federal government to accompany any policy request with long-term cash. The $\$ 700$ million in new operating money recommended by the Naylor report was a bare minimum requirement. This represents only about $15 \%$ of the new short-term health care money announced by the first ministers in September 2004 and about $0.5 \%$ of the country's overall health spending. So far, the federal government has promised a little over $\$ 400$ million. Another billion dollars would be nice.

The next challenge will be to develop the on-the-ground contacts necessary for an effective public health system. Nearly a quarter of the US Centers for Disease Control's 8,600 employees are based outside the head office of Atlanta, Georgia. Most work in state and local public health offices. The Epidemic Intelligence Service provides training for field epidemiology and gives the US an expert "flying squad" of disease control specialists. The CDC also assists with the development and evaluation of public health programs at the state and local level.

In the past, efforts to develop such relationships in Canada have been compromised by the intergovernmental affairs process. But will the new institutional structure of the agency allow it more flexible relationships with other public health organizations? Let us hope that such relationships and programs will not get bogged down by major intergovernmental negotiations.

\section{A mandate as broad as public health itself}

Another challenge to the agency is timeless for public health, "How broad the mandate?" It was concerns about communicable disease that led to the agency's establishment. Often decision-makers seemed confused about public health's other mandates, especially health promotion. The Naylor report recommended a broad role and the agency is subsuming a variety of health promotion programs, including the Community Action Program for Children and the Canadian Prenatal Nutrition Program. However, the agency will be continually challenged to advance these programs within a political environment which too often displays little understanding of a population health approach.

Nineteenth-century public health pioneer Rudolf Virchow claimed that diseases were caused by defects in society. He further argued that governments should prevent disease through economic and social policies. ${ }^{4}$

Public health advocates have historically linked public health with social change and made alliances with social reformers. But, by definition, political and economic elites draw their privileged positions from the status quo. They tend to be disquieted by calls for change, especially if it will cost money.

Public health has used two major approaches to promote social change. The first is to speak out. This is now part of the platform offered to a chief public health officer. A hundred years ago, Toronto medical officer of health, Dr. Charles Hastings, toured reporters through slum housing to prod the politicians to make housing reforms.Mr. Martin assured reporters that he would support Dr. Butler-Jones' independence. However, the whole public health community will have to practice eternal vigilance to preserve this liberty.

Public health's second approach to social change has been to partner with citizens and their organizations. The Toronto public health department partnered with the South Riverdale Community Health Centre to clean up a major lead pollution problem. This partnership also led to new policies at national and international levels.

Unfortunately, the weakening of the public health infrastructure has compromised public health's abilities to work with communities. At the same time, community health centres have developed an approach which combines primary health care with public health services. In addition, almost all regional health authorities are looking at new primary health care models which integrate direct service (often to vulnerable groups) with prevention. While some projects are mainly focussed on medical care, others (e.g. in Saskatoon ${ }^{5}$ ) have an explicit public health focus.

New policies in Quebec have removed the 147 CLSCs from direct community control, but the centres continue to provide a community infrastructure for public health work. They are an important component of Quebec's population health assessment process and its response to public health emergencies. The new public health agency would find its work easier in other provinces if there were similar networks.

\section{CONCLUSION}

The federal government has a major opportunity to reform public health in Canada. With enabling legislation, it has established a new Public Health Agency of Canada and appointed its first chief public health officer. Current circumstances are more favourable for public health than for many years. However, governance and legislation is only one aspect of reform. Public health advocates will need to continue the pressure to ensure adequate funding, new cooperative federal-provincial decisionmaking, and a broad focus for public health work.

\section{REFERENCES/REFERENCES}

1. Naylor CD. Learning from SARS - Renewal of Public Health in Canada. October 2003. A report prepared for the federal Minister of Health. See: http://www.hc-sc.gc.ca/english/ protection/warnings/sars/learning.html (Accessed October 7, 2003).

2. Public Health Agency of Canada website: http://www.phac-aspc.gc.ca/about_apropos/ federal_strategy_e.html\#pillar3 (Accessed October 3, 2004).

3. Federal Department of Finance Fiscal Monitor July 2004. http://www.fin.gc.ca/FISCMON/2004-07e.html (Accessed October 4, 2004).

4. Taylor R, Rieger A. Medicine as social science: Rudolf Virchow on the typhus epidemic in Upper Silesia. Int J Health Serv 1985;15:547-59.

5. See Saskatoon Health Region website: http://www.saskatoonhealthregion.ca/your health/ps_primary_health.htm (Accessed October 4, 2004). 
ÉDITORIAL

\section{Pour faire progresser la santé publique au Canada}

\author{
Michael M. Rachlis, MD, MSc, FRCPC
}

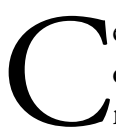
es dernières années, le SRAS, l'ESB et d'autres flambées de maladies transmissibles ont révélé le manque d'attention accordée à la santé publique au Canada. Selon le rapport fédéral sur le SRAS publié en 2003 sous l'égide du D David Naylor, les spécialistes avertissaient le gouvernement des problèmes de la santé publique depuis des années, mais on a fait peu de cas de leurs mises en garde. Par conséquent, écrit le $\mathrm{D}^{\mathrm{r}}$ Naylor, « il y avait beaucoup à apprendre de la crise du SRAS au Canada - en grande partie du fait que de nombreuses autres leçons n'avaient pas été prises en compte auparavant ${ }^{1}$.»

Dans ce numéro de la RCSP, le $\mathrm{D}^{\mathrm{r}}$ Kumanan Wilson de l'Université de Toronto soulève d'importantes questions sur la gouvernance de la santé publique au Canada. Depuis que le $\mathrm{D}^{\mathrm{r}}$ Wilson nous a soumis son article, le gouvernement canadien a créé l'Agence de santé publique du Canada et nommé son premier agent de santé publique en chef. Faisons donc le point sur les événements des six derniers mois, en expliquant ce que nous devons faire pour progresser.

\section{Une nouvelle agence de santé publique pour le Canada}

Dans le budget fédéral de mars 2004, Ottawa a consenti un financement important à une nouvelle Agence de santé publique du Canada, et le ministre des Finances a promis que d'autres fonds seraient disponibles à l'avenir. En septembre 2004, l'Agence a officiellement vu le jour. Son administration centrale se trouve à Winnipeg, mais elle aura aussi des bureaux à Ottawa. Elle sera rattachée aux autres services de santé publique du pays par l'intermédiaire de six centres de collaboration nationaux et d'un réseau de santé publique pancanadien dont la mise en place est prévue pour bientôt. En attendant, le site Web de l'Agence de la santé publique indique que ce réseau pourrait « servir de complément et, s'il s'avère efficace, prendre en charge certains des mécanismes et arrangements actuels de collaboration intergouvernementale ${ }^{2} »$. Il est également suggéré que le réseau serve de forum pour :

- assurer un dialogue soutenu sur les questions de santé publique;

- coordonner les interventions d'urgence en santé publique;

- élaborer des stratégies nationales de santé publique;

- encadrer l'élaboration de normes et d'ententes nationales notamment en matière de partage des ressources et des données, et d'accréditation des professionnels de la santé;

- appuyer la mise en place de centres d'expertise en santé publique à travers le pays.

Le 24 septembre 2004, le premier ministre a annoncé la nomination du $\mathrm{D}^{\mathrm{r}}$ David ButlerJones, de la Saskatchewan, au poste de premier agent de santé publique en chef. Comme le rapport Naylor le recommandait, la nouvelle agence est indépendante de Santé Canada. Le $\mathrm{D}^{\mathrm{r}}$ Butler-Jones relèvera directement du ministre fédéral de la Santé, l'honorable Ujjal Dosanjh. Ainsi, l'Agence pourra passer des marchés directement avec les organismes de santé publique provinciaux et locaux. Pour recruter et administrer son personnel, elle aura aussi une politique de ressources humaines souple, semblable à celles du secteur privé.

Les libéraux sont maintenant à la tête d'un gouvernement minoritaire et devront donner suite à leurs promesses. Comme la croissance économique a dépassé les prévisions budgétaires, l'argent ne devrait pas manquer ${ }^{3}$. Et compte tenu des réformes de la santé publique en cours en Ontario et dans d'autres provinces, les circonstances sont très propices à l'élaboration de politiques de santé publique au Canada. Cependant, l'efficacité de la nouvelle agence

Professeur agrégé au Département de gestion et d'évaluation des politiques sanitaires de l'Université de Toronto, 13 Langley Avenue, Toronto (Ontario) M4K 1B4, Tél. : (416) 466-0093, Courriel : michaelrachlis@rogers.com dépendra des aléas de la politique tout autant que des modalités de gouvernance fixées dans les toutes nouvelles mesures législatives et réglementaires fédérales.

\section{Qui est responsable de la santé publique au Canada?}

Comme l'indique le $\mathrm{D}^{\mathrm{r}}$ Wilson, la santé publique est une compétence fédérale et provinciale à la fois. Malheureusement, jusqu'à tout récemment, le gouvernement fédéral a très peu fait valoir ses droits dans ce domaine.

Selon la Constitution, les quarantaines, la loi pénale et le commerce interprovincial et international sont du ressort du gouvernement fédéral. La Constitution l'autorise aussi à voter des lois pour assurer "la paix, l'ordre et le bon gouvernement". Il est vrai, comme l'indique le $\mathrm{D}^{\mathrm{r}}$ Wilson, que la portée concrète de ces pouvoirs est ambiguë. Mais comme pour tous les pouvoirs, ceux-ci risquent de s'atrophier s'ils ne sont jamais utilisés.

Le gouvernement fédéral bénéficie également d'un pouvoir de dépenser. Les tribunaux l'ont confirmé : il peut disposer de ses fonds comme il l'entend, même dans des domaines de compétence provinciale comme les soins de santé ou les services sociaux. Sans ce pouvoir de dépenser, il n'existerait pas de régime d'assurancemaladie au Canada.

Enfin, les traités et accords internationaux conclus par le gouvernement fédéral lui conferent une autorité considérable. Durant la crise du SRAS, l'Organisation mondiale de la santé (OMS) a transigé directement avec le gouvernement fédéral, sans passer par les provinces ou les organismes locaux de santé publique. L'alerte aux voyageurs publiée par l'OMS concernait Toronto, mais elle a eu des conséquences économiques majeures dans tout le pays. Notre pays a beau tolérer une certaine confusion dans les mandats de santé publique, ce n'est pas le cas des organismes internationaux. Et les événements ont finalement forcé les décideurs canadiens à s'occuper des dysfonctions du réseau de la santé publique.

\section{Que devons-nous faire pour aller de l'avant?}

Toutes ces mesures de réforme initiales sont encourageantes, mais les défenseurs de la santé publique devront veiller au grain pour qu'elles soient effectivement mises en œuvre. 
La première priorité doit être l'accroissement des ressources fédérales. En théorie, le gouvernement fédéral possède toute la marge de manœuvre constitutionnelle voulue pour jouer un rôle vigoureux en santé publique, mais en pratique, il doit fonctionner avec les provinces et par leur intermédiaire.

C'est en partie en comprimant ses paiements de transfert aux provinces que le gouvernement fédéral a résolu ses problèmes de déficit. Vingt ans de compressions fédérales imposées par trois administrations successives ont créé un climat de méfiance entre Ottawa et les provinces - au point où celles-ci exigent maintenant du gouvernement fédéral qu'il accompagne toute demande en matière de politiques d'un financement à long terme. Les nouveaux fonds de fonctionnement de 700 millions de dollars recommandés dans le rapport Naylor n'étaient que le strict minimum. Ils ne représentent qu'environ $15 \%$ des nouvelles dépenses à court terme en soins de santé annoncées par les premiers ministres en septembre 2004, et environ $0,5 \%$ des dépenses globales du pays pour la santé. Jusqu'ici le gouvernement fédéral a promis un peu plus de 400 millions de dollars. Un milliard de plus ne ferait pas de mal.

Le défi suivant sera de tisser, sur le terrain, les liens nécessaires à un réseau de santé publique efficace. Près du quart des 8600 employés des Centres de contrôle américains des maladies (CDC) sont en poste à l'extérieur de l'administration centrale d'Atlanta, en Géorgie. La plupart travaillent pour les organismes de santé publique des municipalités et des États. L'Epidemic Intelligence Service leur offre une formation en épidémiologie d'intervention, ce qui procure aux États-Unis une équipe volante de spécialistes du contrôle des maladies. Les CDC contribuent aussi à l'élaboration et à l'évaluation des programmes de santé publique à l'échelle des États et des municipalités.

Par le passé, les efforts déployés pour établir des liens semblables au Canada se sont coincés dans le mécanisme des affaires intergouvernementales. La nouvelle structure institutionnelle de l'Agence lui donnerat-elle la marge de manœuvre nécessaire dans ses rapports avec les autres organismes de santé publique? S'il s'avère que ces rapports et ces programmes exigent des négociations intergouvernementales élaborées, les choses risquent de traîner en longueur.

\section{Un mandat aussi vaste que la santé publique}

La nouvelle Agence a un autre défi à relever, l'éternel défi de la santé publique, celui de déterminer l'envergure de son mandat. L'Agence est née de la crainte des maladies transmissibles. Souvent, les décideurs semblent décontenancés par les autres mandats de la santé publique, surtout la promotion de la santé. Conformément aux recommandations du rapport Naylor, qui voulait donner un vaste rôle à l'Agence, celle-ci prend en charge plusieurs programmes de promotion de la santé, dont le Programme d'action communautaire pour les enfants et le Programme canadien de nutrition prénatale. Toutefois, l'Agence aura le défi constant de faire progresser ces programmes dans un climat politique où se manifeste trop souvent une ignorance de l'approche axée sur la santé de la population.

Au XIX ${ }^{e}$ siècle, le pionnier de la santé publique Rudolf Virchow faisait valoir que les maladies étaient causées par des défauts dans la société, et que les gouvernements devaient prévenir ces maladies par le biais de politiques économiques et sociales ${ }^{4}$.

Les défenseurs de la santé publique ont depuis longtemps associé celle-ci au changement social et conclu des alliances avec les réformateurs de la société. Mais par définition, les élites politiques et économiques tirent leur position privilégiée du statu quo. Les appels au changement suscitent donc des craintes chez eux, surtout si les mesures préconisées coûtent de l'argent.

Les intervenants en santé publique ont deux grands outils pour favoriser le changement social. Le premier consiste à s'exprimer. Cela se fait maintenant grâce à la tribune accordée aux directeurs de la santé publique. Il y a 100 ans, le médecinhygiéniste torontois Charles Hastings avait organisé à l'intention des journalistes une visite guidée des taudis pour pousser la classe politique à adopter des réformes en matière de logement. M. Martin a assuré aux journalistes qu'il soutiendra l'indépendance du $\mathrm{D}^{\mathrm{r}}$ Butler-Jones, mais toute la collectivité de la santé publique devra faire preuve d'une vigilance constante pour préserver sa liberté d'action.

Le second outil de changement social utilisé par les intervenants de la santé publique consiste à s'associer aux citoyens et à leurs regroupements. Le Service de santé publique de Toronto, par exemple, s'est associé au centre de santé communautaire de South Riverdale pour nettoyer une importante source de pollution par le plomb. Et cette association a donné lieu à de nouvelles politiques aux paliers national et international.

Malheureusement, l'affaiblissement des infrastructures en santé publique a réduit la capacité de collaboration des intervenants avec les collectivités. En parallèle, les centres de santé communautaire ont donc mis au point une approche qui combine soins de santé primaires et services de santé publique. De plus, presque tous les offices régionaux de la santé se tournent vers de nouveaux modèles de soins de santé primaires qui intègrent les services de première ligne (souvent axés sur les groupes vulnérables) et la prévention. Certains projets assurent principalement des soins médicaux, mais d'autres (p. ex., à Saskatoon ${ }^{5}$ ) ont un mandat de santé publique explicite.

Au Québec, de nouvelles politiques ont retiré aux 147 CLSC leur contrôle communautaire direct, mais ces centres demeurent des infrastructures communautaires qui favorisent les efforts en santé publique. Ils sont un élément important du processus québécois d'évaluation de la santé de la population et de réaction aux urgences de santé publique. La tâche de la nouvelle Agence de la santé publique serait facilitée s'il existait des réseaux semblables dans les autres provinces.

\section{CONCLUSION}

Les circonstances sont particulièrement propices à une réforme de la santé publique au Canada. Grâce à des mesures législatives, le gouvernement fédéral a créé une nouvelle Agence de santé publique du Canada et nommé son premier agent de santé publique en chef. La situation n'a pas été aussi favorable à la santé publique depuis de nombreuses années. Mais la gouvernance et la législation ne sont qu'un aspect de la réforme. Les défenseurs de la santé publique devront continuer à faire pression pour obtenir un financement suffisant, un nouveau processus décisionnel fédéral-provincial concerté et un vaste mandat pour les interventions en santé publique. 